\title{
L'institution du divin et du collectif ou les politiques du réel de la prédication évangélique
}

Instituting the Divine and the Collective, or the Politics of Reality in Evangelical Preaching

\section{Philippe Gonzalez}

\section{OpenEdition Journals}

\section{Édition électronique}

URL : http://journals.openedition.org/edc/365

DOI : $10.4000 /$ edc.365

ISSN : 2101-0366

\section{Éditeur}

Université Lille-3

\section{Édition imprimée}

Date de publication : 1 décembre 2006

Pagination : 53-67

ISBN : 2-9514961-7-6

ISSN : $1270-6841$

\section{Référence électronique}

Philippe Gonzalez, «L'institution du divin et du collectif ou les politiques du réel de la prédication évangélique », Études de communication [En ligne], 29 | 2006, mis en ligne le 20 novembre 2014, consulté le 20 avril 2019. URL : http://journals.openedition.org/edc/365 ; DOI : 10.4000/edc.365

Ce document a été généré automatiquement le 20 avril 2019

(c) Tous droits réservés 


\section{L'institution du divin et du collectif ou les politiques du réel de la prédication évangélique}

Instituting the Divine and the Collective, or the Politics of Reality in Evangelical Preaching

Philippe Gonzalez

\section{[...] car le mot est prononcé ; et ce petit mouvement des lèvres suffit à ébranler l'univers. \\ Louis Lavelle, La parole et l'écriture ${ }^{1}$}

L'évangélisme est une forme de protestantisme relativement peu connue en France, voire méconnue. Parmi les travaux sociologiques s'étant penchés sur ce phénomène religieux, rares sont ceux qui ont opté pour une perspective phénoménologique ou praxéologique, laissant ainsi dans l'ombre une part essentielle et constitutive de ce que signifie concrètement "être un évangélique». Le présent article se propose de montrer la pertinence d'une approche sensible aux actes de langage pour investiguer l'évangélisme, et cela à partir d'un moment central de sa pratique: une prédication. L'article est structuré autour de quatre interrogations. Comment une approche ethnographique et discursive de la religion est-elle en mesure de restituer la spécificité de son objet, notamment de rendre compte des phénomènes de mise en présence des membres avec la divinité ? Quels sont les actes de langage qui composent une prédication? Comment s'articulent institution de la présence divine et institution du collectif? Comment user analytiquement de la notion de performatif lorsque cet usage va à l'encontre de la compréhension indigène des membres - en d'autres termes, qu'en est-il d'une description désengagée? 


\section{L'approche : une ethnographie de la coprésence}

2 Il n'est pas lieu de discuter ici les définitions sociologiques habituelles de l'évangélisme². Notons simplement qu'elles se révèlent généralement pauvres pour décrire l'expérience des membres, notamment leur rapport à la divinité. Ce rapport est principalement médiatisé par la Bible, ouvrage que les évangéliques tiennent pour la Parole de Dieu. C'est pourquoi, l'approche ethnographique, et en particulier l'analyse d'une prédication, va dévoiler tout autre chose que le maigre « usage orthodoxe ou littéral » des Écritures que supposent bien des études sociologiques. On verra, au contraire, la mise en coprésence d'un auditoire et d'une divinité au travers de la médiation offerte par une prédication prenant appui sur le texte biblique. À l'invisible d'une improbable définition sociologique, l'analyse va substituer le visible d'engagements mutuels, et en particulier d'un Dieu qui s'engage vis-à-vis de ses fidèles ${ }^{3}$. Or ces engagements sont apprêtés, rappelés et réitérés lors de l'ensemble cérémoniel que constitue le culte dominical. D'où l'intérêt d'une approche ethnographique de la célébration, afin de mettre en lumière les modalités de donation, à la fois spécifiques et ordinaires, du phénomène religieux ${ }^{4}$.

3 L'élément central d'un culte évangélique est la prédication, celle-ci représentant l'équivalent de l'eucharistie en régime catholique. S'il est inconcevable pour des catholiques de célébrer une messe sans eucharistie ${ }^{5}$, se passer de communion ne pose pas de difficulté majeure à des évangéliques. Par contre, l'impensable, pour ces derniers, serait un culte sans "message", celui-ci représentant le moment fort du service dominical. Alors que le catholicisme discerne la présence réelle du Christ dans l'eucharistie, les évangéliques envisagent le prêche comme le moment de la proclamation de la Parole de Dieu, soit comme la saturation maximale de la présence divine lors de la célébration ${ }^{6}$. Dès lors, afin de penser l'ordinaire de la rencontre entre le divin et l'assemblée, il convient de scruter le moment du sermon et les opérations déployées par les membres afin de ressentir le présence divine. Une saisie ethnographique de cet objet est d'autant plus aisée que les assemblées ont pour coutume d'enregistrer, sur un support audio qu'on peut facilement acquérir, si ce n'est l'ensemble du culte, du moins la prédication. Cet enregistrement répond à des besoins internes à la communauté, permettant notamment aux absents d'écouter le service. Recueilli sur le terrain au cours des cinq dernières années, mon corpus contient une vingtaine de prédications durant chacune entre trente et cinquante minutes.

\section{La prédication : du texte (biblique) à la Parole (de Dieu)}

4 La richesse des données et le format du présent article empêchent une restitution approfondie de la prédication. Dès lors, le parti pris analytique consistera à présenter différents moments d'une prédication, afin de dégager la saillance du travail opéré par le prédicateur et ses auditeurs dans la transformation du texte biblique en la voix du divin. Le terme "analyse » convient assez peu de sorte qu'il vaudrait mieux opter pour un expression moins ambitieuse, telle "passage en revue». Bien que des éléments analytiques soient mobilisés, on fera l'économie d'une bonne part de la démonstration. Celle-ci s'appuie sur une attention fine aux éléments séquentiels, catégoriels et énonciatifs sollicités par l'orateur. Quant à la conservation des marques d'oralité dans la 
transcription, elle restitue des indicateurs énonciatifs importants, prévenant ainsi le rabattement trop rapide sur la sémantique des énoncés que serait susceptible d'entraîner une transcription plus lisse.

Trois moments de la prédication vont être passés en revue: (a) une séquence initiale donnant l'interprétant du message; (b) la lecture biblique sur laquelle s'appuie et s'organise le propos; (c) la prière conclusive du prédicateur, transférant les propriétés de l'actant « David» sur les auditeurs et amenant la présence divine à saturation?

\section{L'interprétant de la prédication}

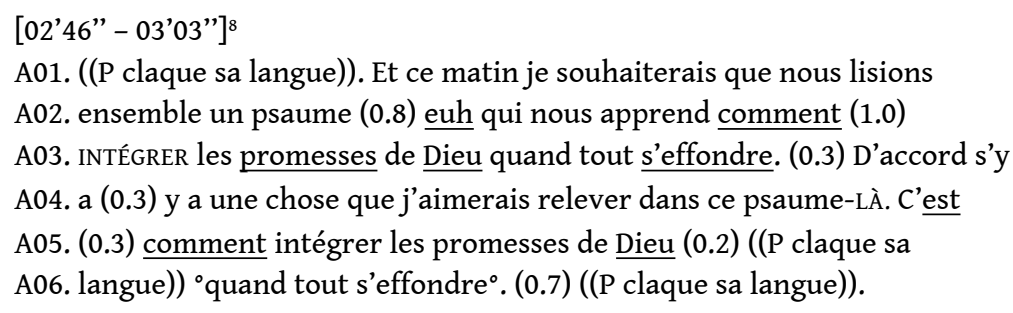

Le prédicateur $[\mathrm{P}]$ débute son message par une plaisanterie narrant les mésaventures d'un perroquet. Le récit produit l'effet escompté, le rire de l'auditoire, permettant à l'orateur d'introduire le sujet de son message : « comment intégrer les promesses de Dieu quand tout s'effondre». Les modulations dans l'élocution du prêcheur portent sur des termes renvoyant à cette même thématique [A02-03]. La lecture du psaume et son interprétation à venir sont présentées comme contenant la solution pour les auditeurs. L'usage du déictique temporel "ce matin » couplé à l'idée d'une lecture collective fait entrevoir l'horizon d'un apprentissage commun à l'égard de l'agir divin. Ainsi, le passage par la plaisanterie est une manière habile d'introduire le sujet de la prédication et d'indiquer le support biblique à partir duquel une solution sera énoncée. L'ensemble du message est à saisir comme la résolution de la tension entre les difficultés auxquelles s'affronte l'existence humaine et les promesses divines. Finalement, ce texte fait entendre à l'auditoire, envisagé comme un "nous " renvoyant à un "être évangélique», des recommandations que Dieu lui adresse expressément, malgré la distance qui sépare la rédaction du psaume de ses auditeurs actuels. L'orateur (de même que ses destinataires) envisage la Bible comme une ressource permettant de régler des problèmes concrets. L'interprétant qu'il donne à son message indique et autorise implicitement les herméneutiques ordinaires que les membres seraient susceptibles de déployer, rapportant leurs situations existentielles à la réalité du texte biblique, afin d'y discerner le secours de Dieu.

\section{La lecture du Psaume 3}

[05'20" - 06'43"]

B01. Psaume TROIs Psaume de David quand il fuyait devant son fils Absalom.

B02. (0.9) ((P claque sa langue)) (0.2) Éternel (0.5) qu'ils sont nombreux

B03. mes adversaires. (0.7) NOMBREUX ceux qui se lèvent contre moi. (0.7)

B04. NOMBREUX CEUX qui disent à mon sujet $(0.2)$ POINT DE SALUT pour lui auprès

B05. de Dieu ! (1.0)

B06. ${ }^{\circ}$ Mais toi Éternel ${ }^{\circ}(0.3)$ tu es pour MoI un bouclier. $(0.7)^{\circ} \mathrm{Tu}$ es ma

B07. gloire ${ }^{\circ}(0.2)^{\circ}$ et tu relèves ma tête ${ }^{\circ} .(0.8)^{\circ}$ À haute voix ${ }^{\circ}(0.3)^{\circ} \mathrm{je}$

B08. crie à l'Éternel ${ }^{\circ}(0.7)^{\circ}$ et il me répond ${ }^{\circ}(0.6)^{\circ}$ de sa montagne

B09. sainte $^{\circ}$. (1.8) 
B10. ${ }^{\circ} \mathrm{Je}$ me couche ${ }^{\circ}(0.6)^{\circ}$ et je m'endors ${ }^{\circ}$. (0.7) Je me réveille car

B11. l'Éternel ${ }^{\circ}$ me soutient ${ }^{\circ}$. (0.8) JE NE CRAINS PAS les myriades de gens qui

B12. de toutes parts $(0.6)^{\circ}$ se sont mis contre moi ${ }^{\circ}$. (1.2)

B13. Lève-toi Éternel (0.2) sauve-moi mon Dieu ! (0.9) Car tu frappes à la

B14. joue tous mes ennemis. (0.7) Tu brises les dents des méchants. (0.7) À

B15. l'Éternel est le salut. (0.7) Que ta bénédiction (0.4) ${ }^{\circ}$ soit sur ton

B16. peuple ${ }^{\circ}$. (1.2)

B17. ((P claque sa langue et compulse ses notes))

B18. (2.5)

B19. Ce matin j'aimerais qu'on - on regarde (0.2) le constat que fait David

B20. qui est un constat d'un monde hostile. (0.3) On REGARDERA ensuite le

B21. réconfort (0.8) que Dieu offre. C'est un Dieu protecteur. (0.3) On

B22. regardera en troisièmement - en troisième point le résultat $(0.4)$ le

B23. repos que Dieu donne. (0.4) Et enfin le (0.4) dernier point le recours

B24. que nous avons (0.4) quand justement tout s'effondre. (0.7)

7 Après une brève explication sur le genre littéraire du psaume de lamentation, le prédicateur s'attelle à la lecture du texte qui servira de base à son message [B01-16]. Entre-temps, les auditeurs ont eu loisir de trouver le passage dans leurs exemplaires personnels de la Bible et suivent attentivement la lecture qui leur est faite. Les inflexions de la voix de l'orateur (visuellement restituées par la transcription) indiquent à son public des éléments saillants sur lesquels le propos du message va s'appuyer. Vient ensuite une explicitation du plan de la prédication, celui-ci s'organisant autour de quatre points déjà dégagés par les pauses du lecteur et auxquels il confère des titres [B19-24]: (a) le constat de David d'un monde hostile [B01-05, 19-20]; (b) le réconfort offert par Dieu [B06-09, 20-21]; (c) «le repos que Dieu donne»[B10-12, 22-23]; et finalement, (d) «le recours que nous avons » en Dieu [B14-16, 23-24]. On voit dès lors de quelle manière le découpage séquentiel du texte biblique constitue une ressource permettant au prédicateur d'organiser le cours de sa prédication.

8 La présentation des quatre points met en lumière une autre facette du mouvement accompli par le sermon. Au premier mouvement, propre à l'agencement du texte et à son déroulement - second mouvement - dans la temporalité de la prédication, vient s'en ajouter un troisième, allant de "David» au «nous» formé par les auditeurs et le prédicateur. Ce transport amène avec lui le réconfort, le repos et le recours de Dieu, c'està-dire l'assurance de sa présence aux côtés de ceux qui traversent aujourd'hui l'adversité. Ce dernier mouvement condense les opérations déployées par l'herméneutique ordinaire, soit un passage du symbolique du texte à l'indiciel d'une présence, du passé de l'écrit à l'actualité de la Parole, restituant les modalités d'un rapport oraculaire à la Bible.

\title{
Prière conclusive
}

\author{
[36'31" - 37'18'] \\ C01. (1.1) \\ C02. On prie? \\ C03. (2.1) \\ C04. Seigneur quelle joie pour nous que de savoir (0.7) que tu es vivant \\ C05. (1.1) \\ C06. au milieu des orages (0.7) des tourmentes même (0.2) des tourments que \\ C07. nous avons $(0.4)^{\circ}$ parfois suscités par nos propres péchés ${ }^{\circ}$. (1.5) \\ $\mathrm{C} 08$. Tu es vivant $(0.3)$ et tu es $(0.7)^{\circ}$ celui qui relève notre tête ${ }^{\circ}$. (0.8) \\ C09. Tu es ${ }^{\circ}$ le bouclier qui s'interpose ${ }^{\circ}$. (0.7) Tu es la ${ }^{\circ}$ gloire $^{\circ}(0.9)^{\circ} \mathrm{de}$ \\ C10. notre vie ${ }^{\circ}$. (0.8) Et nous te remercions que le jour ${ }^{\circ}$ vient $^{\circ}(1.0)$
}


C11. où Seigneur nous serons $\underline{\text { dans }}$ la ${ }^{\circ}$ gloire ${ }^{\circ}(0.2)^{\circ}$ auprès de toi ${ }^{\circ}(1.1)$

C12. et où toutes ces choses (0.6) pâliront ${ }^{\circ}$ totalement ${ }^{\circ}$. (1.2)

C13. Donne à cette assemblée et à chacun d'entre nous Seigneur la $(0.4)$

C14. la grâce de pouvoir nous abreuver de tes promesses (0.5) ${ }^{\circ}$ au milieu de

C15. toutes les circonstances de l'existence que nous pouvons rencontrer ${ }^{\circ}$.

La prédication s'achève sur une prière de l'orateur. Celle-ci cristallise le passage du texte biblique à la voix divine en attribuant pleinement les propriétés - et donc, la réalité - du psalmiste à l'auditoire. La plupart des participants a refermé sa Bible. Les yeux clos, la tête inclinée, ils participent à la prière en acquiescant à certains de ses propos par un léger balancement du buste, un marmonnement ou un « oui, Seigneur » chuchoté. Que Dieu soit "vivant", est une condition nécessaire à sa présence, celle-ci se faisant ressentir dans la protection que la divinité accorde aux auditeurs et se déclinant suivant le triple motif davidique du bouclier, de la gloire et du relèvement de la tête [C08-10]. Vient ensuite un remerciement à propos de la consommation eschatologique de toutes choses, consommation qui débouche sur la perspective d'une coprésence immédiate et continuelle avec la divinité. Mais en attendant, cette présence transite par les " promesses » divines à l'égard de « cette assemblée ", des promesses rendues disponibles par une pratique oraculaire du texte biblique.

Le survol analytique a montré les actions conduisant du texte à la voix, depuis un rapport symbolique à la Bible jusqu'à l'expérience de la présence et de l'agir divins, et cela au travers du surgissement d'une Parole. Dès lors, il est possible de poser trois thèses. Les premières sont catégorielles: (a) le texte biblique fournit aux membres un cadre symbolique permettant de faire sens de leur pâtir (et de leur agir) ; (b) le texte biblique permet aux membres de faire sens de l'identité et de l'agir des autres. La dernière thèse comporte une dimension causale: (c) pour tout auditeur (Ar) catégorisé au moyen d'un texte biblique, s'il est perçu comme partageant certaines propriétés d'un actant (At) du récit, alors l'action de Dieu (Dr) à son égard peut être similaire, dans le monde vécu, à celle dont est l'objet l'actant de la part de la divinité dans le récit (Dt). Les conditions de félicité de ce mouvement analogique allant du texte à l'auditoire sont garanties par l'ancrage historique du récit biblique, le fait qu'il y ait eu un précédent garantissant la possibilité du déploiement d'un agir divin similaire hic et nunc.

\section{La double institution du divin et du collectif}

11 L'analyse a montré le montage séquentiel de la prédication dans la production d'une coprésence. Il serait réducteur de faire reposer l'entière efficacité du dispositif sur le travail du prédicateur, comme s'il imposait automatiquement cette présence à son auditoire. De fait, l'orateur configure une place que ses destinataires sont invités à venir occuper : le premier propose une structure formalisée de sens que les seconds pourront investir. La prière finale ne constitue que l'aboutissement de cette proposition d'identification. Cependant, cette cristallisation excède la simple invitation à l'identification: elle instaure une réalité où la divinité prend part à l'existence des humains (fidèles, en particulier) et les accompagne dans l'épreuve. À ce point, il vaut la peine d'explorer le concept de performatif, afin de dégager, dans le mouvement, ses potentialités heuristiques.

Lorsque J. Austin (1970) met au point la notion d'énoncé performatif, c'est en premier lieu pour montrer que la constatation ne représente de loin pas, à elle seule, le paradigme discursif du langage ordinaire. L'opposition tranchée entre performatif et constatif donnera 
lieu à des développements successifs plus nuancés où cette opposition ne constitue que le cas limite d'une théorie plus générale de l'illocution, soit d'une pragmatique. Du coup, c'est l'ensemble des énoncés qui se voit doté de la triple dimension locutoire (le fait de dire), illocutoire (ce qu'on fait en disant) et perlocutoire (ce qui est suscité chez autrui par le dire). Le cas du baptême représente l'illustration habituelle de l'énoncé performatif : pour que l'acte remplisse ses conditions de félicités, un énonciateur autorisé énonce la formule à la première personne du singulier « Je te baptise... ». Par contre, la prédication qui nous occupe - et cela s'étend à l'ensemble de mon corpus - n'exhibe pas de marqueurs syntaxiques ou lexicaux permettant de déceler immédiatement la présence d'un performatif. Lors de sa prière, le prédicateur énoncé, sur un mode constatif, l'évidence de la présence de Dieu et de son agir. Il n'en demeure pas moins que le dispositif mobilisé au cours du prêche donne lieu à la mise en coprésence avec la divinité, et donc, fait advenir quelque chose. Par conséquent, on se trouve bien face à une institution performative d'un réel saturé par le divin.

Cette institution du divin va de pair avec l'institution d'un collectif particulier, celui des membres à même d'effectuer les actions que le prédicateur sollicite de leur part, dans le passage du texte à l'audition de la Parole. Non seulement leur compétence est engagée dans le montage des éléments de la prédication, mais leur assentiment est nécessaire, afin de garantir la pertinence du sermon. Pour cela, chaque auditeur doit (a) opérer le montage pour soi, et (b) percevoir la validité du montage pour tous. En d'autres termes, les membres prennent part simultanément à l'ordonnancement séquentiel du message et à son bon ordre. C'est ici le socle conventionnel sur lequel repose l'institution performative du divin, qui, en ayant lieu, produit réflexivement le collectif qui l'institue.

Une dernier élément mérite d'être signalé : les membres ne prêtent pas attention aux opérations qu'ils mettent en œuvre afin d'instituer le divin et le collectif. Ils ne se soucient que du résultat du montage, celui-ci apparaissant rétrospectivement comme un réel ayant toujours déjà été : «Tu es vivant [...] celui qui relève notre tête [...] le bouclier qui s'interpose [...] la gloire de notre vie »[C08-10]. Le produit de la prédication évangélique partage ainsi d'importantes affinités avec les modalités de la référence scientifique. Dans les comptes-rendus de découvertes, les médiations ayant permis de transformer l'indexation locale d'un échantillon, d'une mesure ou d'un événement sont oblitérées par les chercheurs afin de garantir le transport spatio-temporel du phénomène investigué . Les médiations s'effacent pour installer un rapport (envisagé comme) direct à l'objet et produire sa stabilité ontologique. De manière similaire, dans la prédication, l'ancrage référentiel du récit biblique constitue la plateforme permettant le déplacement et l'irruption d'un agir divin, ici et maintenant, tout en pourvoyant à son assise ontologique $^{10}$.

\section{Des politiques du réel antagonistes ou les difficultés d'une description désengagée}

La description de la prédication sur le mode du performatif s'achoppe pourtant à une triple difficulté : (a) elle ne tient pas compte du fait que les membres possèdent une conception propre des performatifs; (b) elle risque de réduire l'expérience phénoménologique des participants engagés aux procédures séquentielles déployées par le sermon en tant qu'acte de langage ; (c) elle menace d'endosser une conception neutre de la description, dans l'illusion d'un rapport transparent au langage. 
16 La première nuance à apporter quant à une description usant de la catégorie performatif tient à la capacité des évangéliques à discriminer entre certains types d'actes langagiers. Les membres possèdent un classement propre pour évaluer la force illocutoire de propos ritualisés. À leurs yeux, une prière de conversion constitue le paradigme par excellence du performatif - généralement, elle est dite à la première personne par le converti qui a témoigné de sa foi auprès de l'inconverti, ce dernier répétant intérieurement ou à haute voix ce que lui dit le premier. La formule baptismale constitue une autre forme d'énoncé performatif. Cependant, peu de membres seraient susceptibles d'acquiescer à une analyse qui verrait dans la prière conclusive ci-dessus un énoncé performatif, au sens où il produirait ce qu'il dit. Cette réticence est une conséquence logique du caractère conventionnel, et donc socialement indexé, des actes de langage. En d'autres termes, l'analyste n'a pas à décréter si tel énoncé est doté d'une force illocutoire, mais se doit de décrire la portée pragmatique d'une énonciation, suivant les effets reconnus par, et induits chez, les membres

17 Le second écueil serait celui d'une description réductionniste du fait religieux. En posant le caractère opératoire, effectif en soi, d'une classe d'énoncés, l'analyste serait tenté de se débarrasser de l'épineuse difficulté d'une transcendance qui affleurerait dans ses données, en réduisant l'expérience du divin par le membre au produit d'un montage séquentiel effectué par ce même membre. De là, il n'y a qu'un pas vers une sociologie critique dénonçant l'illusion de l'individu pris dans les mirages métaphysiques engendrés par ses propres usages langagiers. Or, selon M. de Certeau (1990), un tel mouvement analytique tend à oublier que si le langage peut informer, au sens de transmettre des informations, il n'informe pas forcément, au sens d'imposer une forme. Le prédicateur met à disposition une forme que les membres viendront investir, dans un montage collaboratif. Dès lors, les actes langagiers appellent à un remplissage phénoménologique pouvant ménager un espace pour la visitation divine : le tout de l'expérience ne se laisse pas subsumer sous l'explicitation d'un dire.

Les critiques précédentes annoncent la troisième, relative au problème posé par une description désengagée. Si la force illocutoire d'un énoncé dépend de son ancrage conventionnel, il est nécessaire, afin de saisir sa portée, d'être engagé dans l'activité même où s'inscrit cet énoncé et qu'il vient modifier. Comment, dès lors, produire une description de notre prédication qui aille le plus loin possible dans une restitution du phénomène et rende justice à l'expérience des membres ? Cette difficulté ne peut être résolue qu'en partant des effets pragmatiques de la prédication elle-même. Ainsi, au terme de sa prière, le prédicateur rend compte d'une réalité qui n'est pas immédiatement habitable pour le sociologue et la plupart de ses lecteurs. Cette réalité résiste, obligeant l'étranger que je suis à divers types de rationalisation (l'explication réductionniste renvoyant à la magie du performatif constituerait, par exemple, une issue confortable, signalant un deus ex machina à défaut de reconnaître une présence divine). Cependant, si l'on évite la réduction, la résistance du phénomène nous rappelle à son institution, soit au fait que les membres participent activement à sa mise en place, de manière conventionnelle, par leur engagement, tout comme nous concourons à l'établissement d'autres régimes de réalité où la divinité n'intervient pas dans la causalité du monde. 


\section{La résistance du réel comme prise sur le phénomène}

19 À ce point, nous nous achoppons à des politiques du réel ${ }^{11}$ antagonistes. Dans une perspective durkheimienne, cet antagonisme représente la résistance propre du fait social et l'accès qu'il nous offre à son intelligibilité (Durkheim, 1990). Quand bien même les membres ne réserveraient pas un bon accueil à une lecture en termes de performatifs, ils verraient dans notre incapacité à saisir ou à souscrire à la prière du prédicateur le signe de notre possible extériorité en regard du collectif évangélique. Ainsi, la perception du phénomène se trouve incluse dans l'orientation de l'activité : c'est l'engagement dans les opérations déployées par le collectif qui rend disponible l'expérience de la divinité, et simultanément, la rend indisponible à une description désengagée. La description qui se limite à une caractérisation performative du sermon construit - et s'adresse à - un collectif désengagé (un lectorat de sociologues, par exemple), au risque de réduire l'expérience religieuse à un montage pragmatique et de perdre le phénomène. Car ce que la prédication institue, c'est un monde appelant à une autre forme d'habitation; s'y engager, c'est rendre saillante une discontinuité avec d'autres mondes dépourvus de ce type de transcendance. Toutefois, cette saillance est perceptible comme une altérité pour celui qui est extérieur à la communauté, venant altérer son expérience du réel, et donc l'engageant sur un autre niveau. Si je ne peux ressentir une visitation, je suis en mesure d'en ressentir l'absence. Dès lors, la prière conclusive de notre prédicateur ne constitue pas simplement un exposé factuel sur un monde habité par le divin, mais engendre simultanément la possibilité - et l'impossibilité - d'un engagement.

\section{BIBLIOGRAPHIE}

Austin, J. L., (1970), Quand dire, c'est faire, Paris, Seuil, [1962].

Bastian, J.-P., (dir.), (2004), La recomposition des protestantismes en Europe latine. Entre émotion et tradition, Genève, Labor et Fides.

Certeau De, M., (1990), L'invention du quotidien, I : Arts de faire, Paris, Gallimard.

Claverie, É., (2003), Les guerres de la Vierge. Une anthropologie des apparitions, Paris, Gallimard.

Durkheim, É., (1990), Les règles de la méthode sociologique, Paris, P.U.F., [1895].

Fath, S., (2005), Du ghetto au réseau. Le protestantisme évangélique en France 1800-2005, Genève, Labor et Fides.

Garfinkel, H. et al., (1981), « The Work of a Discovering Science Construed with Materials from Optically Discovered Pulsar », Philosophy of the Social Sciences, Vol. 11, 131-158.

Gonzalez, P., (2005), Herméneutiques ordinaires : les protestantismes évangéliques comme expérience collective, Genève, Conférence Universitaire de Suisse Occidentale, DEA en sociologie sous la dir. de O. Tschannen \& J. Widmer. 
Harding, S. F., (2000), The book of Jerry Falwell : Fundamentalist Language and Politics, Princeton, Princeton University Press.

Latour, B., (1990), « Quand les anges deviennent de bien mauvais messagers », Terrain, Vol. 14, 76-91.

Latour, B., (1993), « Le topofil de Boa-Vista. La référence scientifique : montage photophilosophique », in : B. Conein et al., Les objets dans l'action, Paris, EHESS, coll. « Raisons pratiques ", $\mathrm{n}^{\circ} 4,187-216$.

Lavelle, L., (2005), La parole et l'écriture, Paris, Éditions du Félin, [1942].

Marion, J.-L., (2005a), Étant donné. Essai d'une phénoménologie de la donation, Paris, P.U.F., [1997].

Marion, J.-L., (2005b), Le visible et le révélé, Paris, Cerf.

Piette, A., (1999), La Religion de près. L'activité religieuse en train de se faire, Paris, Métailié.

Piette, A., (2003), Le fait religieux. Une théorie de la religion ordinaire, Paris, Economica.

Pollner, M., (1975), « ‘The Very Coinage of Your Brain' : The Anatomy of Reality Disjunctures », Philosophy of the Social Sciences, Vol. 5, $n^{\circ} 4,411-430$.

Stromberg, P. G., (1993), Language and Self-transformation. A Study of the Christian Conversion Narrative, Cambridge, Cambridge University Press.

\section{NOTES}

1. (Lavelle, 2005 : 46) Je remercie Ch. Monnot, J. Stavo-Debauge et les lecteurs anonymes de la revue pour leurs commentaires judicieux.

2. La plupart des spécialistes en sciences religieuses définissent les évangéliques par quatre traits: (a) rapport orthodoxe ou littéral à la Bible, (b) centralité (de la mort) du Christ, (c) nécessité d'une conversion personnelle, (d) propension à l'évangélisation (Bastian, 2004 ; Fath, 2005). J'ai eu l'occasion de me livrer ailleurs à une critique systématique de ces catégories (Gonzalez, 2005). Pour des approches plus pragmatiques de l'évangélisme, voir S. Harding (2000) ou P. Stromberg (1993).

3. Ce qui apparaîtra clairement lors de la prière conclusive du prédicateur.

4. Ma démarche s'inscrit dans le fil du «théisme méthodologique » pratiqué par É. Claverie (2003) et A. Piette (2003). L'idée centrale est la suivante : l'homme religieux se sait impliqué dans la construction de l'interaction avec les entités surnaturelles, tout en demeurant conscient de l'autonomie de ces dernières; c'est pourquoi, il est impératif de saisir cette mise en coprésence au risque de passer à côté de la dimension ordinaire du fait religieux.

5. Voir à ce sujet la très belle ethnographie d'A. Piette (1999) mettant en évidence les difficultés inhérentes à la pénurie des vocations et au palliatif que représente l'instauration des assemblées dominicales en absence de prêtre (ADAP).

6. Le format de l'article me contraint à quelques raccourcis, notamment sur ce point. L'énoncé est vrai pour les évangéliques de type "classique », et en particulier «fondamentalistes »; il devrait par contre être nuancé pour la tendance charismatique, celle-ci connaissant des phénomènes d'inhabitation par l'Esprit sous la forme de parlers en langues, de prophéties ou de guérisons.

7. La spécificité de mon objet et mes choix sémantiques me font voisiner avec la phénoménologie de J.-L. Marion, en particulier ses travaux sur les phénomènes « saturés » et les modalités de la « donation » du divin (Marion, 2005a; b). 
8. Les chiffres entre crochets indiquent le minutage de la prédication. Les conventions de transcription sont les suivantes : MAJUSCULE pour un volume élevé ; ${ }^{\circ}$ points ${ }^{\circ}$ pour un volume bas ; soulignement pour une modulation de la voix ; $(0.5)$ pour les pauses en secondes; (parenthèses) pour une transcription incertaine; ((parenthèses doubles)) pour une action non verbale.

9. Je pense ici aux travaux de B. Latour (1993) sur le topofil ou à ceux de H. Garfinkel et alii (1981) sur la découverte d'un pulsar.

10. Contrairement à ce qu'avance un $B$. Latour (1990) soucieux de distinguer de manière étanche entre une logique de procession (religieuse) et logique de réseau (scientifique), la référence religieuse (en régime évangélique) ne dissocie précisément pas le signifiant et le signifié, «les anges ne deviennent [pas] de bien mauvais messagers ". Il n'y aurait pas, d'un côté, un accès rigoureux au lointain par une extension contrôlée dans l'espace-temps, et de l'autre, une mise en présence par un retour sur le hic et nunc. L'embrayage sur la présence divine nécessite justement l'ancrage référentiel. Pour un traitement approfondi de la référentialité, voir Gonzalez (2005).

11. Je reprends l'expression de M. Pollner (1975) tout en lui conférant un sens plus « politique », c'est-à-dire en explicitant ses implications dans la formation des collectifs.

\section{RÉSUMÉS}

Le présent article montre l'importance d'une approche attentive aux actes de langage pour rendre compte $d u$ phénomène religieux que constitue l'évangélisme. Partant du survol analytique d'une prédication, on montre comment s'organise pragmatiquement l'expérience de la divinité par les membres et la clôture du collectif. Cette démonstration permet un retour réflexif sur la pratique de la description sociologique, l'enjeu consistant à éviter une réduction rapide de l'expérience phénoménologique du divin aux cadres pragmatiques qui la rendent possible.

This paper uses an approach that underlines the importance of speech-acts in order to shed light on the religious phenomenon known as evangelicalism. Based on the analytical overview of a sermon, it is shown how the members' experience of the divine and the establishment of group boundaries are organized on the pragmatic level. Such a methodology allows for a self-critical look at the practice of sociological description in order to avoid an all-too rapid reduction of the phenomenological experience of the divine to the pragmatic framework which enables it.

\section{INDEX}

Mots-clés : actes de langage, prédication évangélique

Keywords : speech acts, evangelical preaching

\section{AUTEUR}

PHILIPPE GONZALEZ

Philippe Gonzalez prépare actuellement une thèse de doctorat sur les protestantismes évangéliques et s'attache à décrire les liens entre l'expérience religieuse, la constitution d'un 
collectif ecclésial et les rapports que ce dernier entretient à un espace public politique. Outre la sociologie des religions, l'auteur développe une sociologie ancrée dans la phénoménologie et la pragmatique. Son premier livre est à paraître courant 2007 et s'intitule Herméneutiques ordinaires : l'évangélisme comme expérience collective. Adresse électronique : philippe.gonzalez@unifr.ch. 